\title{
EMI Due to Electric Field Coupling on PCB
}

\author{
M.H. Pong, C.M. Lee and X. Wu \\ Department of Electrical \& Electronic Engineering, Hong Kong University \\ Pokfulam Road, HONG KONG \\ Fax : +852-25598738, Phone : +852-28597099 \\ E-mail : mhp@hkueee.hku.hk
}

\begin{abstract}
In switching converter circuits, EM noise can couple between PCB traces through the effect of electric field coupling. An experiment using a flyback converter verifies the severity of this effect. Further experiments and field plots confirm that a good PCB layout can significantly reduce conducted EMI due to unintentional E-field coupling.
\end{abstract}

\section{INTRODUCTION}

Engineers in the field of switch mode power supply (SMPS) often encounter the problem of electromagnetic interference (EMI). The switching nature of SMPS make it an inevitable source of Electromagnetic Interference (EMI). More and more countries have established regulations against products that generate EMI. Engineers and researchers are striving to reduce EMI by various methods.

From experience it is well known that circuit layout design on a printed circuit board (PCB) plays a very important part in reduction of EMI. A good circuit layout can very often solve EMI problems without any changes in circuit topology or components in the circuit. However, there is very little information available about the EMI performance of a SMPS until the circuit is fully laid out on a PCB and tested. If EMI issues are ignored until a problem is revealed by testing or in normal operation, EMI fixes tend to be applied at the test or even production stages of product development, which can lead to solutions that are unsatisfactory, unnecessarily expensive, or both.

Every EMI problem comprises three elements: a source, a receiver and a mechanism by which noise is coupled from the source to the receiver as in Fig.1. A source refers to a noise generator with high $\mathrm{dv} / \mathrm{dt}$ or $\mathrm{di} / \mathrm{dt}$, and the receiver refers to a victim circuit or EMI measurement equipment.

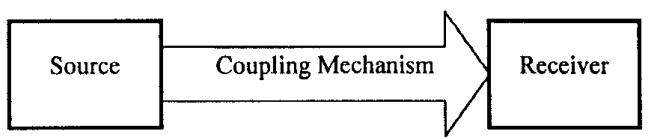

Fig.1. Basic Elements in EMI Problems

Coupling mechanism can be categorized into conducted coupling and non-conducted coupling (Fig.2). Conducted coupling refers to interference along a conducting path that links up the source and the receiver.
The effect is often suppressed by circuit isolation or filtering. Non-conducted coupling comprises of far field coupling and near field coupling. Far field is also known as antenna effect by which noise radiates meters away from the source. Near field includes electric field coupling and magnetic field coupling in close vicinity.

In SMPS, electric field coupling has considerable contribution to overall EMI especially those circuits with high voltage transients. In practice, power electronics designers use filter and circuitry approach to suppress EMI. However, near field electric coupling between PCB traces in these circuits may by-pass filters. This paper proposes an alternative approach to analyze such noise coupling effect. Electric field coupling between PCB traces is carefully examined. Experiments on a flyback converter circuit confirm the severity of near field coupling. Electric field emission from PCB traces with elementary shapes are measured and examined. The flyback converter is then re-layout after considering the effect of E-field coupling. Significant reduction in conducted noise is observed.

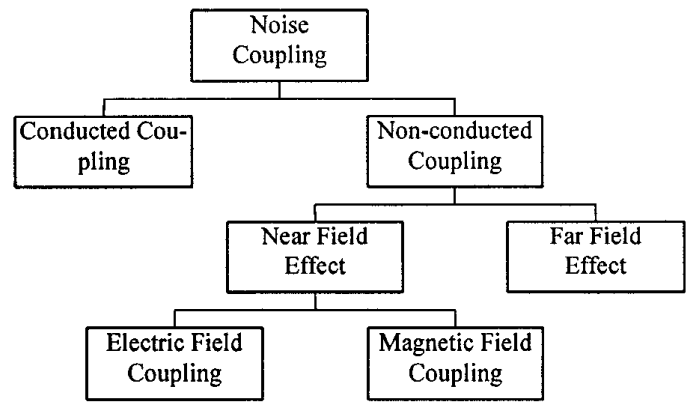

Fig.2. Classification of Noise Coupling Mechanism

\section{VerifiCATION OF EleCtRiC Field Coupling On PCB}

A typical switch mode power supply contains four basic building blocks as shown in Fig. 3 :

1. input filters and rectifier;

2. high-frequency inverter;

3. output section;

4. control circuit.

The input filters and rectifier rectifies and smoothes the a.c. voltage input from the power source. The voltage is then fed to the high frequency inverter by which the line voltage is chopped to a square pulse 
train and the period of pulses is controlled by a switching transistor. A transformer steps down the pulsating voltage to predefined level before going to be the output section. The output section comprises of rectifiers and filters of various topologies, the major function of which is to convert the pulsating voltage to a d.c. voltage.

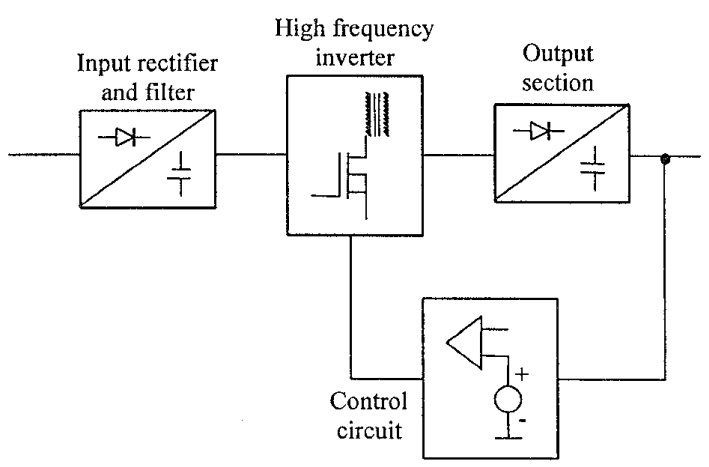

Fig.3. Basic Building Blocks of SMPS

An experiment setup is constructed to verify the severity of near field electric coupling. In this experiment, a typical flyback converter is chosen for EMI measurement. The flyback converter comprises the four basic building block of a SMPS and the circuit design is shown in Fig.4.

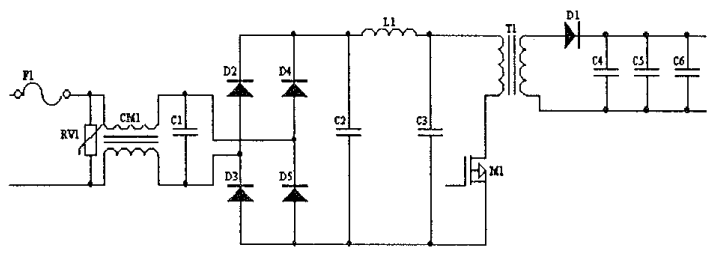

Fig.4. Schematic of Flyback Converter

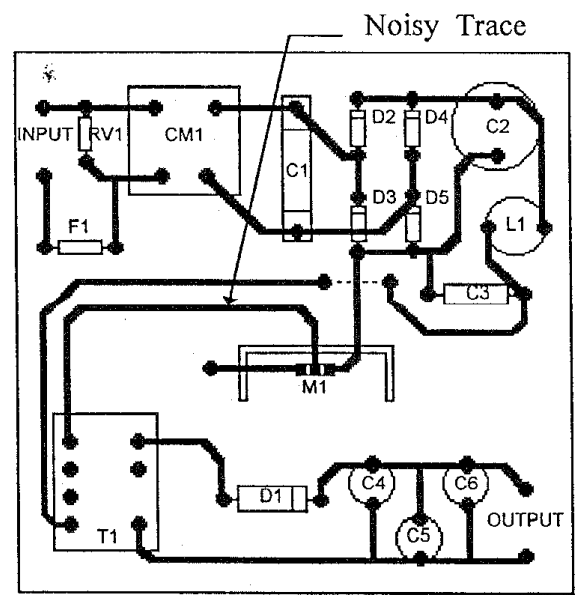

Fig.5. PCB Layout of Flyback Converter
The control block is separated from the power circuit because the low voltage controller is considered as a victim instead of a source. A screened cable links the power transistor and the control circuit. The PCB layout is constructed in a $100 \mathrm{~mm} \times 100 \mathrm{~mm}$ square board as shown in Fig. 5 .

The experiment is set up as shown in Fig.6. A Line Impedance Stabilization Network (LISN) connects between the a.c. mains and the input of the converter. A spectrum analyzer measures the high frequency noise emitted from the converter. This is the standard setup for conducted EMI measurement.

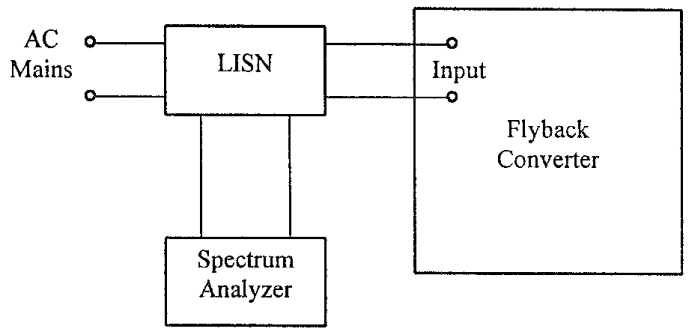

Fig.6. Experimental Setup of Conductor Noise Measurement

Conducted EMI measurement in Fig.8(a) shows the noise emission level exceeds the FCC limit of 48 $\mathrm{dB} \mu \mathrm{V}$ in frequency range $450 \mathrm{kHz}$ to $30 \mathrm{MHz}$.

In the next step the common choke CM1 is removed from the PCB assembly (Fig.7). This leaves an open circuit between the input surge suppresser RV1 and the bridge rectifier. Power is fed into the rectifier directly while the LISN is connected to the input pads as the previous setup. By circuitry approach, an open circuit represents a perfect filter as there is no conducting path for transmission of conducted noise.

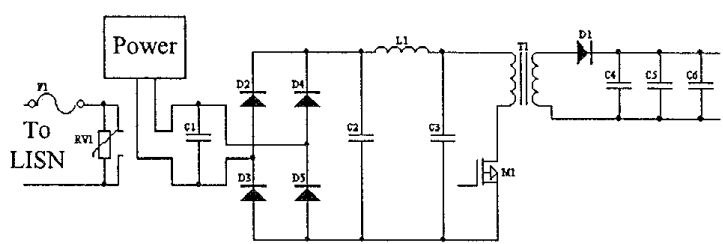

Fig.7. Conducted Noise Measurement after Circuit Isolation

The measurement should be predicted for a very low noise level at the LISN as all conducted noise should have been curbed. However measurement reveals results in a converse way. The noise level is still high and outside the acceptance range of the FCC limit (see Fig.8(b)), although it has dropped compared to the measurement in Fig.8(a).

The noise measured should mainly consist of nonconducted electric field coupled noise. Since the LISN 
is disconnected from the switching circuit, there should be no conducted coupling noise present in the spectrum. The two remaining coupling mechanisms are the categories of far field and near field non-conducted coupling. However the effective range of the far field coupling extends meters away from the circuit. So near field coupling should have contributed a very significant amount. Both electric and magnetic coupled noise appear in the noise spectrum. High $\mathrm{dv} / \mathrm{dt}$ of power devices make electric field the dominate noise source.

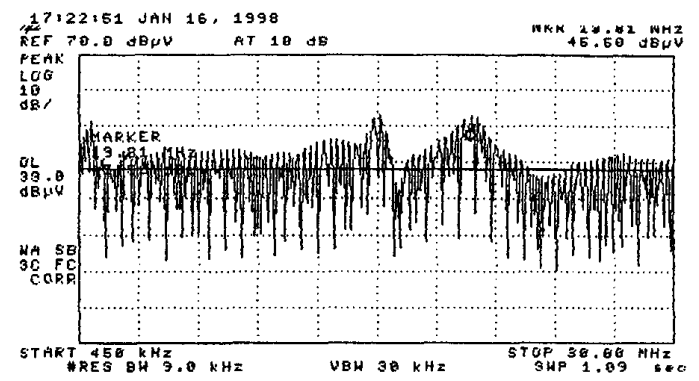

(a) Normal Setup

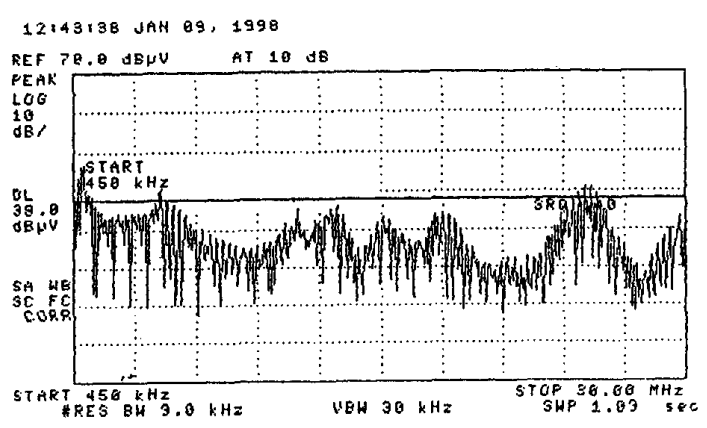

(b) Common Mode Choke is Removed

Fig.8. Spectrum of Conducted Noise Measured

\section{ELECTRIC FIELd COUPLING MECHANISM}

Electric field coupling between PCB traces can be represented by the model [1] shown in Fig. 9. Noise source $\mathrm{Vi}$ is connected to conductor 1 and interference noise couples to conductor 2 through capacitor $C_{12}$. The noise voltage picked up by conductor 2 is represented by

$$
V_{n}=\frac{j \omega\left[\frac{C_{12}}{\left(C_{12}+C_{2 g}\right)}\right]}{j \omega+\frac{1}{R\left(C_{12}+C_{2 g}\right)}} V_{i}
$$

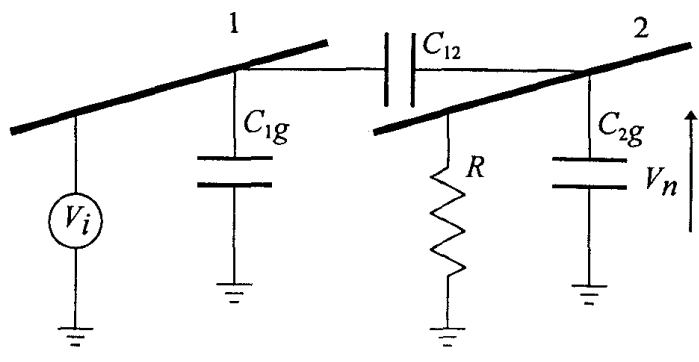

Fig.9. Electric Field Coupling Model

In most practical cases,

$$
R<\frac{1}{j \omega\left(C_{12}+C_{2 g}\right)}
$$

and (1) can be simplified to

$$
V_{n}=j \omega R C_{12} V_{1}
$$

From equation (3) $C_{12}$ is an important factor. It represents the effect of circuit layout in terms of electric field coupling. In practice $C_{12}$ is rather tedious to be derived analytically, especially in complicated $\mathrm{PCB}$ layout. Numerical method is more favorable in calculating the coupling voltage $V_{n}$.

\section{ELECTRIC FIELD PLOTS}

In order to examine the source of electric field in the flyback circuit discussed in section 2, an EMI scanner system is used. The electric field close to the PCB is plotted out and shown in Fig.10. The area of high emission concentrates on the primary side where there is high voltage swing of several hundred volts and high $\mathrm{dv} / \mathrm{dt}$. In particular the trace connecting the drain of the switching MOSFET M1 has high emission [4] and this trace is marked up by an arrow in Fig. 10 .

The "noisy" trace shown in Fig.10 is likely to interfere conducted emission measurement at the power input terminals. In the layout shown in Fig.10, the "noisy " trace is placed rather near to the input terminals. Also it runs over considerable length across the board and is likely to induce electric field coupling. Note that by this layout electric field coupling can bypass the filter elements in the primary circuit and directly links the "noisy trace" and the input terminals.

Once the concept of "noisy" trace is established it is worth looking at emission patterns of PCB traces of elementary shapes. Emission plots of single elementary traces are shown in Figs.11. Each of these traces are excited by a signal source operating at a frequency below $30 \mathrm{MHz}$. At these frequencies the potential along the conductor traces can be considered constant since the signal wavelength is much larger than the length of the trace. 


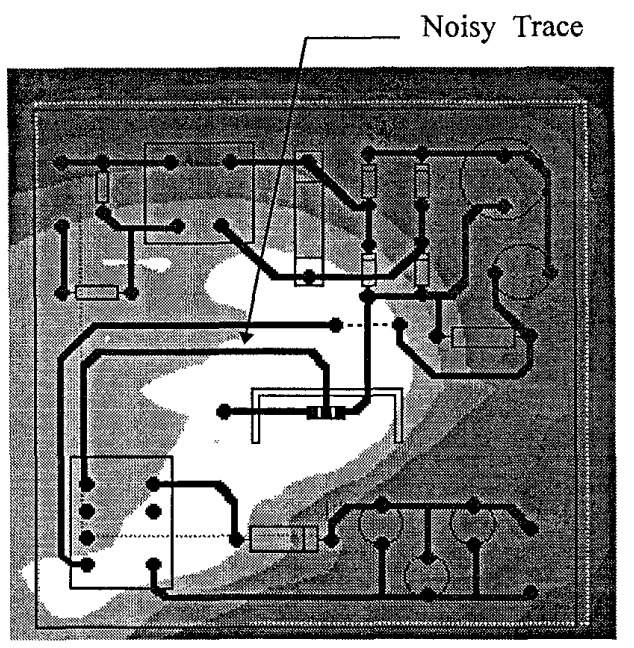

Fig.10. Field Plot of Online Flyback Converter with Noisy Trace Marked Up by an Arrow. Highest reading in white area is $37.88 \mathrm{~dB} \mu \mathrm{V}$ Step between Contour Lines $1.64 \mathrm{~dB} \mu \mathrm{V}$.

Fig.11(a) shows the emission pattern of a single straight conductor. Fig.11(b) shows the pattern of a semi-circular trace, Fig.11(c) shows the pattern of an arc trace and Fig.11(d) shows the pattern of a $L$ shape trace. In each of the plots it can be seen that the Electric field extends over a wide area compared to the dimensions of the trace concerned. One can predict that when another trace is placed in areas of high emission, it is likely to pick up noise due to E-field coupling. Also the shape of PCB trace dictates the pattern of emission. One can choose a suitable traces shape for critical paths, such as traces which carry very large voltage swing. By this way coupling effect can be minimized in advance before the board is been fabricated. Engineers can predict the effect of electric field coupling by observing these plots in PCB design.

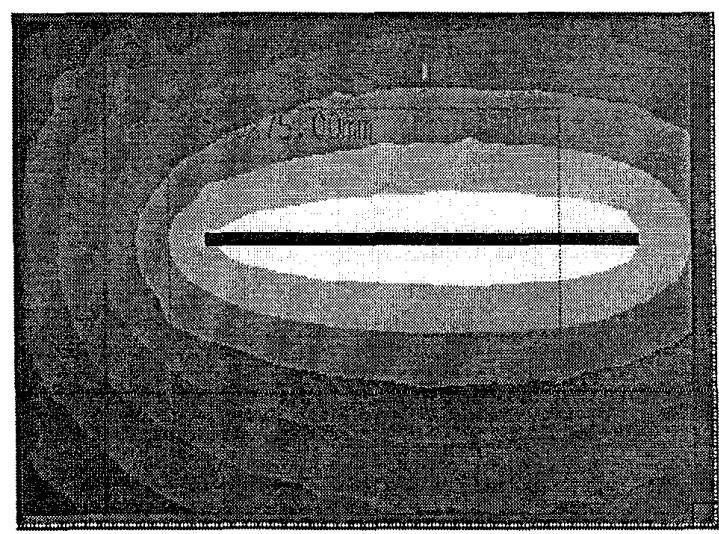

(a) Single Straight Conductor with Trace Width $2 \mathrm{~mm}$ Highest reading in white area is $60.29 \mathrm{~dB} \mu \mathrm{V}$ Steps between Contour Lines $2.20 \mathrm{~dB} \mu \mathrm{V}$

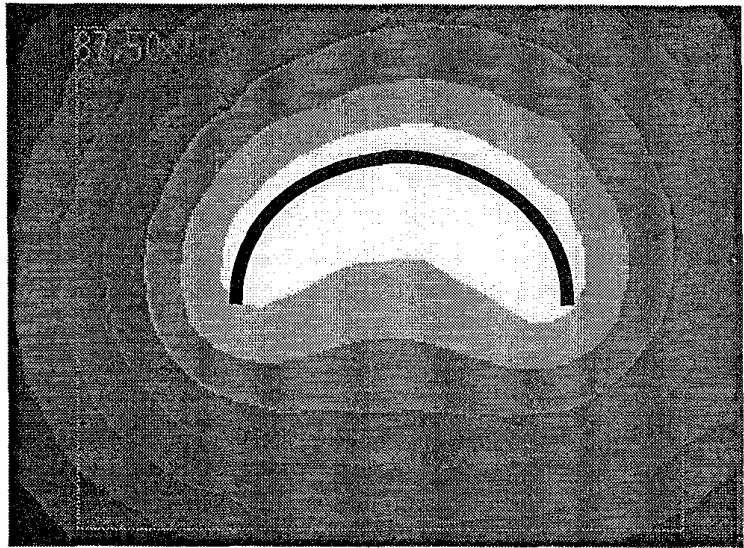

(b) Semi-Circular Conductor with Trace Width $2 \mathrm{~mm}$ Highest reading in white area is $65.76 \mathrm{~dB} \mu \mathrm{V}$ Steps between Contour Lines $1.60 \mathrm{~dB} \mu \mathrm{V}$

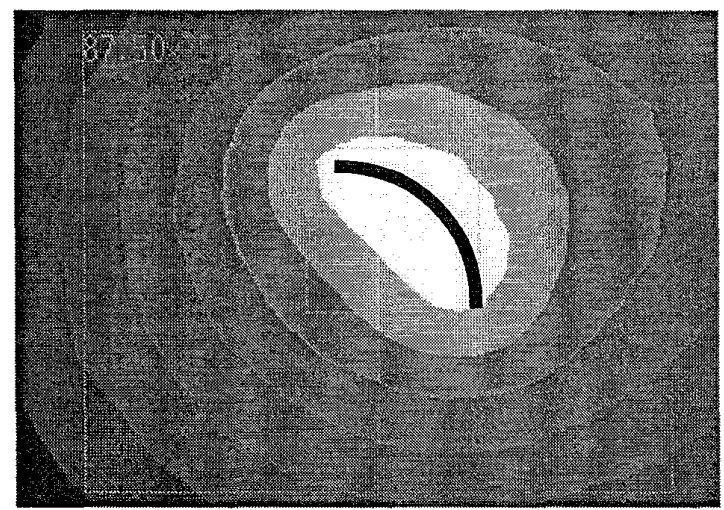

(c) Arc Shape Conductor with Trace Width $2 \mathrm{~mm}$ Highest reading in white area is $64.92 \mathrm{~dB} \mu \mathrm{V}$ Steps between Contour Lines $2.05 \mathrm{~dB} \mu \mathrm{V}$

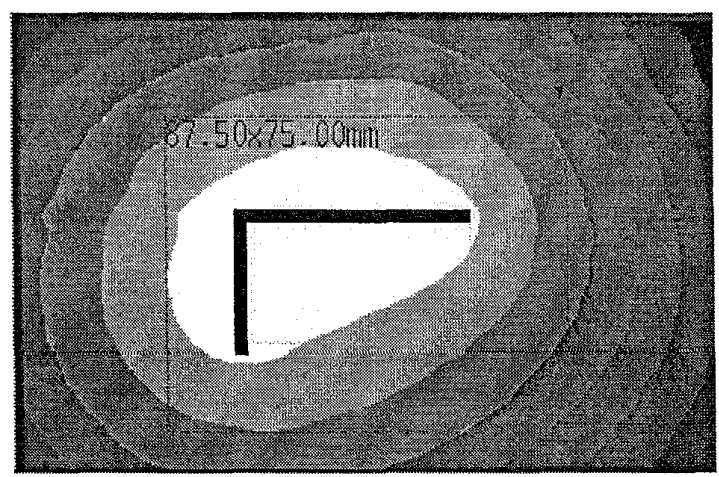

(d) L-shaped Conductor with Trace Width $2 \mathrm{~mm}$ Highest reading in white area is $55.38 \mathrm{~dB} \mu \mathrm{V}$ Steps between Contour Lines $1.40 \mathrm{~dB} \mu \mathrm{V}$

Fig.11. Field Plot of Varies Shape of Traces 


\section{Modifications of THE Flyback Converter LAYOUT}

Experimental results in previous sections verify the importance of E-field coupling on PCB traces. It is also established that "noisy" traces have to be kept away from "receiver" such as input terminals. The shape of a PCB trace dictates its emission pattern.

The layout of the flyback circuit is now modified according to these concepts. It has been identified that the noisy trace is the one connected to the drain of the switching MOSFET. The new PCB is designed to give a greater separation between the noisy trace and the input of the converter which is the victim of electric field coupling as shown in Fig.12. In order to confirm the effect of relocating the trace all component placement remain unchanged with respect to that of Fig.5. This eliminates changes due to structural effects. Also the same components from the previous experiments are used.

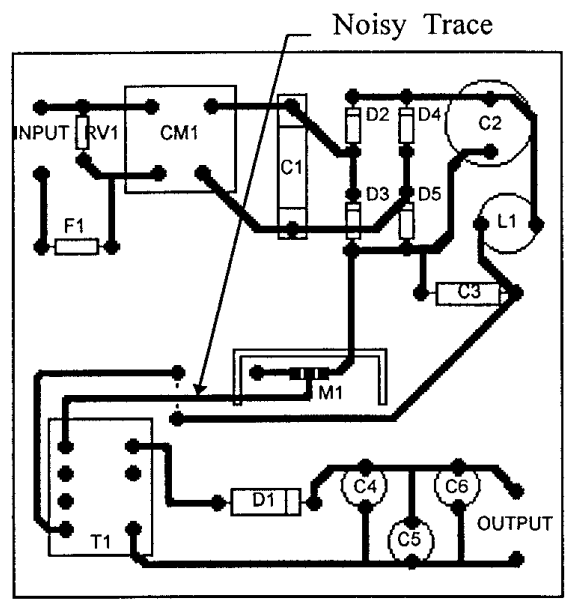

Fig.12. Redesigned PCB Layout of Flyback Converter

The flyback converter with new PCB layout is tested as the same setup of Section 2. The circuit is opened at the location of the common mode choke CM1 so that signals received at the LISN is entirely due to non-conducted coupling.

The noise spectrum is recorded in Fig.13. A comparison of the noise spectrum in Fig. 13 with that of Fig.8(b) reveals that there is nearly $10 \mathrm{~dB}$ drop at frequencies between $450 \mathrm{kHz}$ to $1 \mathrm{MHz}$ and between $10 \mathrm{MHz}$ to $20 \mathrm{MHz}$. This shows clear evidence that trace separation is one of the critical elements affecting EMI emission on the PCB.

The layout is then modified furthermore to reduce noise measured at the input terminals. The MOSFET M1 and the transformer T1 are relocated to the opposite corner of the PCB as shown in Fig.14. The "noisy" trace is hence further separated from the input terminals and its length is greatly shortened.

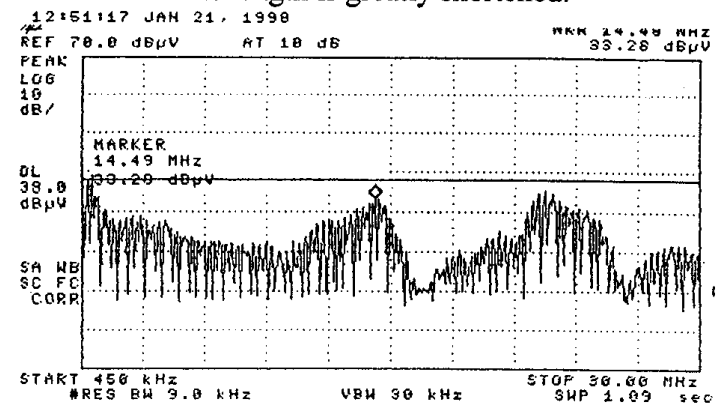

Fig.13. Noise Spectrum of the Flyback Converter with Resigned Flyback Converter

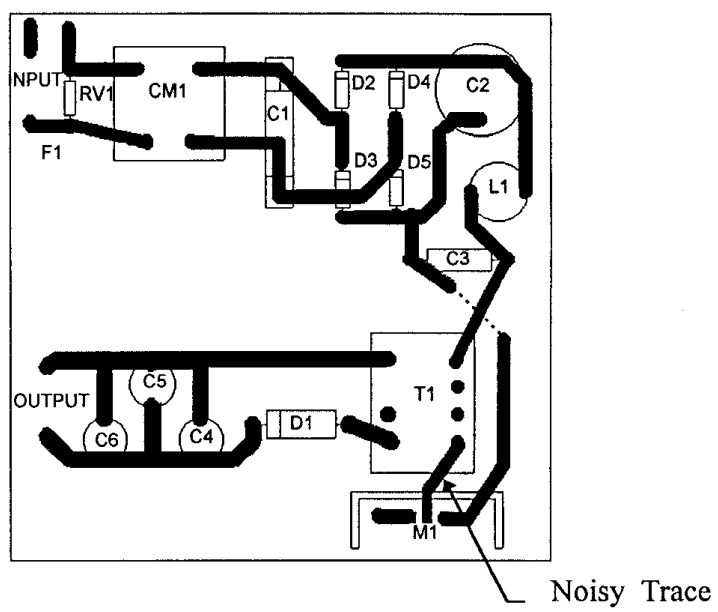

Fig. 14. PCB Layout of Flyback Converter with Further Modifications

The EMI noise spectrum is shown in Fig.15. The spectrum shows significant reduction in noise in comparison with those shown in Fig.13 and Fig.8(b).

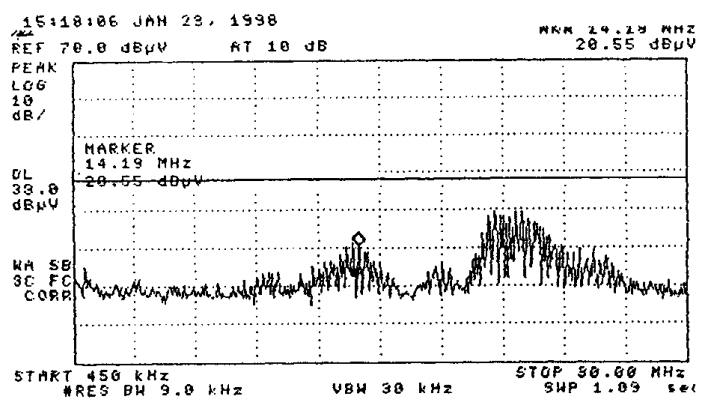

Fig.15. Noise Spectrum of Improved PCB Layout

\section{Conclusions}

Experiments verify the severity of electric field coupling on printed circuit board. It can affect other 
trace without any direct circuit connection. The noise level is high and significant. The noise level can be reduced by careful treatment of noisy traces. This includes increasing trace separation between the noise generating traces and the input traces. Also the shape of trace dictates the emission pattern. These have been verified by significant noise reduction after layout modifications of a flyback converter.

\section{REFERENCES}

[1] C. S. Walker, Capacitance, Inductance and Crosstalk Analysis. Artech House, 1990.

[2] H. W. Ott, Noise Reduction Techniques in Electronic Systems. $2^{\text {nd }}$ Edition, Wiley, New York, 1988.

[3] L. Tihanyi, Electromagnetic Compatibility in Power Electronics. IEEE Press, 1995.

[4] B. M. H. Pong and A. C. M. Lee, "A Method To Measure EMI Due To Electric Field Coupling On PCB", in Proceedings of the PCC 97, 1997.

[5] L. B. Gravelle and P. F. Wilson, "EMI/EMI in Printed Circuit Boards - A Literature Review," IEEE Transactions on Electromagnetic Compatibility, vol. 34, no. 2, May 1992.

[6] J. C. Fluke, Sr., Controlling Conducted Emission by Design. Van Nostrand Reinhold.

[7] C. R. Paul, Introduction to Electromagnetic Compatibility. $1^{\text {st }}$ Edition, Wiley, New York, 1992.

[8] T. N. Bahr and E. J. MacMahon, Electrostatic Discharge Control. Hayden, New York, 1983.

[9] A. N. Faught, "An Introduction to Shield Joint Evaluation using EMU Gasket Transfer Impedance Data," in Proceedings of the IEEE Symposium, pp. 38-44, 1982.

[10] J. Goedbloed, Electromagnetic Compatibility. Prentice Hall, New York, 1990.

[11] R. J. Mohr, "Evaluation Technique for EMI Seams," in Proceedings of the IEEE Symposiumon Electromagnetic Compatibility, pp. 81-84, 1987.

[12] B. Keiser, Principles of Electromagnetic Compatibility, $3^{\text {rd }}$ Edition, Aertech House, Norwood, MA, 1987.

[13] E. D. Knowles and L. W. Olson, "Cable Shielding Effectiveness Testing," IEEE Transaction on Electromagnetic Compatibility, EMC-16, February, 1974.

[14] C. R. Paul and K. B. Hardin, "Diagnosis and Reduction of Conducted Emissions," in Proceeding of the IEEE Symposium on Electromagnetic Compatibility, pp. 19-23, 1988.

[15] C. R. Paul and W. W. Everett III, "Printed Circuit Board Crosstalk," in Proceedings of the IEEE Symposium on Electromagnetic Compatibility, pp. 452-459, 1985.

[16] J. L. N. Violette, D. R. J. White and M. F. Violette, Electromagnetic Compatibility Handbook, Van Nostrand Reinhold, New York, 1987.

[17] D. V. Wood, "The prediction of the EMC Performance of Electronic System Prior to Fabrication", IEEE Transaction

[18] M. K. W. Wu, C. K. Tse and P. Chan, "An Integrated CAD Tool for Switching Power Supply Design with Electromagnetic Interference Prediction", IEEE Transaction

[19] W. John, "Remarks to the Solution of EMC-Problems on Printed-Circuit-Board", IEEE Transaction

[20] K. Nagesware Rao and P. Venkata Ramana, "EMC Analysis in PCB Designs Using and Expert System", IEEE Transaction

[21] M. K. W. Wu and C. K. Tse, "A Review of EMI Problems in Switching Mode Power Supply Design", IEEE Transaction

[22] C. J. Railtion, K. M. Richardson, J. P. McGeehan and K. F. Elder, "The Prediction of Radiation Levels from Printed Circuit Boards by means of the Finite Difference Time Domain Method", IEEE Transaction 